Journal of Theoretical and Applied Mechanics, Sofia, 2016, vol. 46, No. 1, pp. 53-64

\title{
NON-LINEAR ANALYSIS OF MODE II FRACTURE IN THE END NOTCHED FLEXURE BEAM
}

\author{
V. Rizov \\ Department of Technical Mechanics, \\ University of Architecture, Civil Engineering and Geodesy, \\ 1, Chr. Smirnensky Blvd, 1046 Sofia, Bulgaria, \\ e-mail: V_RIZOV_FHE@UACG.BG
}

[Received 08 December 2015, Accepted 29 February 2016]

\begin{abstract}
Analysis is carried-out of fracture in the End Notched Flexure (ENF) beam configuration, taking into account the material nonlinearity. For this purpose, the $J$-integral approach is applied. A non-linear model, based on the Classical beam theory is used. The mechanical behaviour of the ENF configuration is described by the Ramberg-Osgood stress-strain curve. It is assumed that the material possesses the same properties in tension and compression. The influence is evaluated of the material constants in the Ramberg-Osgood stress-strain equation on the fracture behaviour. The effect of the crack length on the $J$-integral value is investigated, too. The analytical approach, developed in the present paper, is very useful for parametric analyses, since the simple formulae obtained capture the essentials of the non-linear fracture in the ENF configuration.

KEY WORDS: Non-linear fracture analysis, beam theory, $J$-integral.
\end{abstract}

\section{Introduction}

The application of laminates has increased significantly in the recent decades, $[1,2,3,4,5,6]$. This is due to high stiffness-to-weight ratio, good processability, and excellent resistance to degradation, which make the laminates a promising alternative to the traditional structural materials.

The strong focus on load-bearing applications leads to a particular attention towards interlaminar fracture behaviour of laminates. Initiation and growth of interlaminar cracks drastically reduce the stiffness and the compressive strength of the laminate and may, in some cases, cause catastrophic failure of the structure. Therefore, the interlaminar fracture continues to attract the 
interest of the researchers $[7,8]$. The fracture is studied usually in terms of strain energy release rate by using the methods of linear-elastic fracture mechanics. The basic assumption of linear-elastic fracture mechanics is that the relation between the strains and the stresses can be expressed by the Hooke's law. However, the applicability of linear-elastic fracture mechanics can be limited by the high fracture toughness (in such cases, the plastic deformation begins prior to macroscopic crack growth). Therefore, the analysis should take into account the material non-linearity $[9,10,11,12,13]$.

Various beam configurations for investigation of interlaminar fracture behaviour have been developed [14, 15, 16, 17, 18, 19, 20, 21]. One of these configurations is the ENF, which is used for characterization of mode II interlaminar fracture [22].

The present paper deals with non-linear fracture analysis of the ENF beam configuration. The Ramberg-Osgood stress-strain curve is used to describe the mechanical response of the ENF. The $J$-integral approach is applied to study the non-linear fracture behaviour. For this purpose, a model based on the Classical beam theory is used. The effect of the material constants in the Ramberg-Osgood equation on the fracture behaviour is analyzed.

\section{Non-linear analysis of mode II fracture}

The present article reports a theoretical study of mode II longitudinal fracture in the ENF beam configuration with taking into account the material non-linearity. The ENF is illustrated schematically in Fig. 1. A longitudinal crack of length $a$ is located in the beam mid-plane. The beam is loaded in

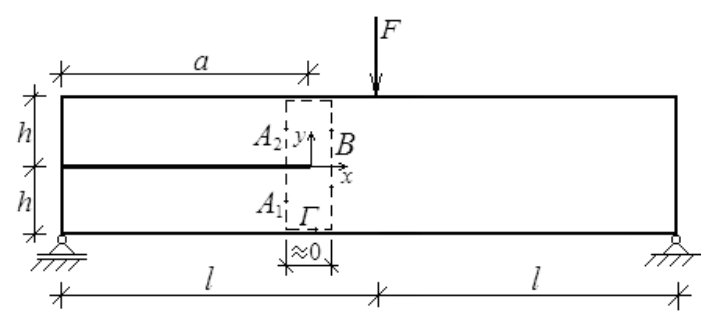

Fig. 1. The ENF beam configuration

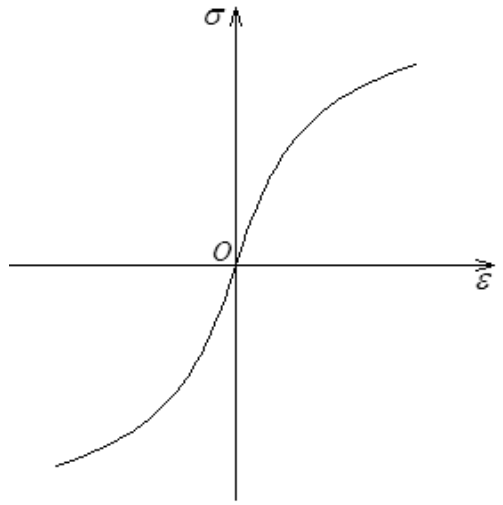

Fig. 2. The Ramberg-Osgood stress-strain curve 
bending by a transverse force, $F$, applied in the mid-span. In this way, mode II crack loading conditions are induced. The beam has a rectangular cross-section of width, $b$, and height, $2 h$.

It was assumed that the mechanical response of the ENF beam configuration follows the Ramberg-Osgood stress-strain curve, which is symmetric with respect to tension and compression as shown in Fig. 2. The stress-strain equation is written as [23]

$$
\varepsilon=\frac{\sigma}{E}+\left(\frac{\sigma}{H_{2}}\right)^{\frac{1}{n_{2}}},
$$

where $\sigma$ is the normal stress, $\varepsilon$ is the linear strain, Eis the modulus of elasticity, $H_{2}$ and $n_{2}$ are material constants.

The $J$-integral approach, developed in [24, 25], was applied here to investigate the non-linear fracture behaviour of the ENF beam configuration. The $J$-integral was expressed as [24]:

$$
J=\int_{\Gamma}\left[u_{0} \cos \alpha-\left(p_{x} \frac{\partial u}{\partial x}+p_{y} \frac{\partial v}{\partial x}\right)\right] d s,
$$

where $\Gamma$ is a contour of integration, going from the lower crack face to the upper crack face in the counter clockwise direction, $u_{0}$ is the strain energy density, $\alpha$ is the angle between the outwards normal vector to the contour of integration and the crack direction, $p_{x}$ and $p_{y}$ are the components of the stress vector, $u$ and $v$ are the components of the displacement vector with respect to the crack tip coordinate system $x y$, and $d s$ is a differential element along the contour $\Gamma$.

In the present study, the $J$-integral was solved over an integration contour, that coincides with the ENF beam cross-sections ahead and behind the crack tip (Fig. 1), i.e. the integration contour has three segments $\left(A_{1}, A_{2}\right.$, and $B)$. Thus, the $J$-integral solution was found by summation:

$$
J=J_{A_{1}}+J_{A_{2}}+J_{B} .
$$

Segments $A_{1}$ and $A_{2}$ coincide with the cross-sections of the lower and the upper crack arm behind the crack tip. Segment $B$ coincides with the ENF beam cross-section ahead of the crack tip (Fig. 1).

The $J$-integral solution in segment $A_{1}$ of the integration contour was obtained in the following way. The components of the $J$-integral were written as:

$$
p_{x}=-\sigma, \quad p_{y}=0,
$$




$$
d s=d z_{1}, \quad \cos \alpha=-1 .
$$

The coordinate, $z_{1}$, varies in the interval $[-h / 2, h / 2]$. The normal stresses and the linear strains distribution in segment $A_{1}$ is reported in Fig. 3.

The strain energy density, $u_{0}$, is equal to the area enclosed by the stressstrain curve $[27,28,29,30]$ (refer to Fig. 4):

$$
u_{0}=\int_{0}^{\varepsilon} \sigma d \varepsilon,
$$

where $d \varepsilon$ can be obtained as [23]:

$$
d \varepsilon=\left[\frac{1}{E}+\frac{1}{n_{2} \sigma}\left(\frac{\sigma}{H_{2}}\right)^{\frac{1}{n_{2}}}\right] d \sigma .
$$

In view of (7), integral (6) was written as:

$$
u_{0}=\int_{0}^{\sigma} \sigma\left[\frac{1}{E}+\frac{1}{n_{2} \sigma}\left(\frac{\sigma}{H_{2}}\right)^{\frac{1}{n_{2}}}\right] d \sigma .
$$

The solution of (8) was found as:

$$
u_{0}=\frac{\sigma^{2}}{2 E}+\frac{\sigma^{\frac{1+n_{2}}{n_{2}}}}{\left(1+n_{2}\right) H_{2}^{\frac{1}{n_{2}}}} .
$$

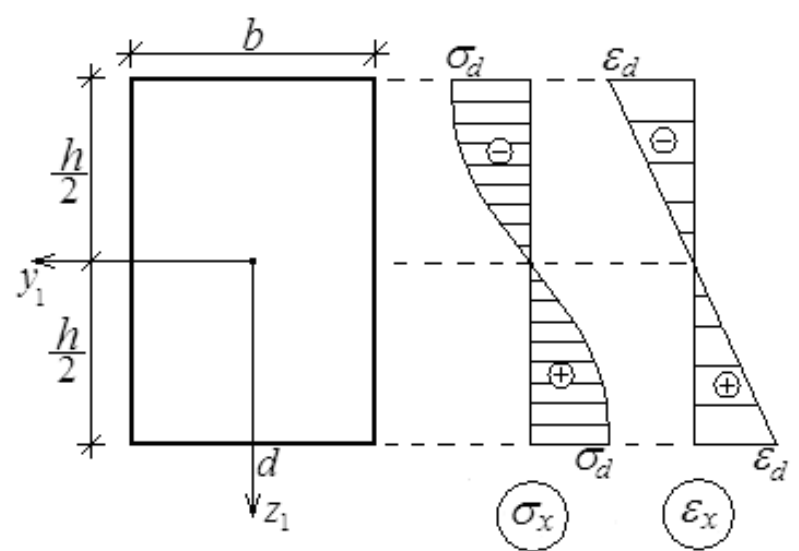

Fig. 3. Diagrams of normal stresses and linear strains in cross-section $A_{1}$ of the lower crack arm 


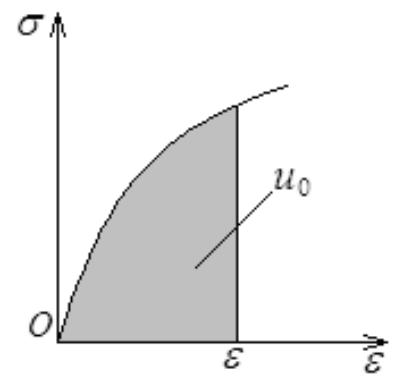

Fig. 4. Strain energy density

The following formula from Mechanics of materials was applied to determine the partial derivative, $\partial u / \partial x$ :

$$
\frac{\partial u}{\partial x}=\varepsilon_{x},
$$

where $\varepsilon_{x}$ was obtained by the Ramberg-Osgood equation, (1). Thus, (10) was rewritten as:

$$
\frac{\partial u}{\partial x}=\frac{\sigma}{E}+\left(\frac{\sigma}{H_{2}}\right)^{\frac{1}{n_{2}}} .
$$

The following relation between $d z_{1}$ and $\varepsilon$ was used in the present analysis [31]:

$$
d z_{1}=\frac{h}{2 \varepsilon_{d}} d \varepsilon,
$$

where $d \varepsilon$ was obtained by (7). The edge linear strain, $\varepsilon_{d}$, was expressed by the Ramberg-Osgood equation:

$$
\varepsilon_{d}=\frac{\sigma_{d}}{E}+\left(\frac{\sigma_{d}}{H_{2}}\right)^{\frac{1}{n_{2}}},
$$

where $\sigma_{d}$ is the normal stress in the cross-section edges (Fig. 3).

The following equation for equilibrium of the cross-section [23] was used here to determine the edge normal stress:

$$
M=2 b\left(\frac{h}{2 \varepsilon_{d}}\right)^{2} \int_{0}^{\sigma_{d}} \sigma\left[\frac{\sigma}{E}+\left(\frac{\sigma}{H_{2}}\right)^{\frac{1}{n_{2}}}\right]\left[\frac{1}{E}+\frac{1}{n_{2} \sigma}\left(\frac{\sigma}{H_{2}}\right)^{\frac{1}{n_{2}}}\right] d \sigma
$$


where $M$ is the crack tip cross-sectional bending moment in the lower crack arm. $M$ was found as (refer to Fig. 1):

$$
M=\frac{F}{4} a .
$$

By substitution of (13) in (14) and solving the integral, we obtained the following algebraic equation with unknown $\sigma_{d}$ :

$$
M=\frac{b h^{2}}{2\left[\frac{\sigma_{d}}{E}+\left(\frac{\sigma_{d}}{H_{2}}\right)^{\frac{1}{n_{2}}}\right]^{2}}\left[\frac{\sigma_{d}^{3}}{3 E^{2}}+\frac{\sigma_{d}^{\frac{1+2 n_{2}}{n_{2}}}}{E H_{2}^{\frac{1}{n_{2}}}} \cdot \frac{1+n_{2}}{1+2 n_{2}}+\frac{\sigma_{d}^{\frac{2+n_{2}}{n_{2}}}}{\left(2+n_{2}\right) H_{2}^{\frac{2}{n_{2}}}}\right]
$$

It can be observed in formula (1), that at $H_{2} \rightarrow \infty$, the RambergOsgood equation transforms into the Hooke's law. This fact was used here to verify equation (16). For this purpose, we substituted $H_{2} \rightarrow \infty$ in (16) and obtained:

$$
\sigma_{d}=\frac{6 M}{b h^{2}} .
$$

Equation (17) coincides with the known relation between the bending moment and the normal stresses in the edges, when a rectangular cross-section deforms in linear-elastic stage, which verifies (16).

Another verification was performed by using the fact that at $E \rightarrow \infty$ and $n_{2}=1$ equation (1) transforms also into the Hooke's law (assuming that the modulus of elasticity is $H_{2}$ ). Indeed, at $E \rightarrow \infty$ and $n_{2}=1$ formula (16) transforms into (17).

Equation (16) was verified also by considering the fact that at $n_{2}=1$ the Ramberg-Osgood equation (1) transforms into the Hooke's law (assuming that the modulus of elasticity is $\left.E H_{2} /\left(E+H_{2}\right)\right)$. By substitution of $n_{2}=1 \mathrm{in}$ (16), we obtained (17), which is also a verification of (16).

By substitution of (4), (5), (9), (11), (12), and (13) in (2) and solving the integral in boundaries from the upper edge normal stress to the lower edge 
normal stress, we found:

$$
\begin{aligned}
J_{A_{1}}= & \frac{h}{2\left(\frac{\sigma_{d}}{E}+\frac{\sigma_{d}^{\frac{1}{n_{2}}}}{H_{2}^{\frac{1}{n_{2}}}}\right)}\left[\frac{\sigma_{d}^{3}}{3 E^{2}}+\frac{\sigma_{d}^{\frac{1+2 n_{2}}{n_{2}}}}{\left(1+2 n_{2}\right) H_{2}^{\frac{1}{n_{2}}} E} \frac{2 n_{2}^{2}+n_{2}+1}{2\left(1+n_{2}\right)}\right. \\
+ & \frac{\sigma_{d}^{\frac{2+n_{2}}{n_{2}}} n_{2}}{\left(2+n_{2}\right)\left(1+n_{2}\right) H_{2}^{\frac{2}{n_{2}}}}-\frac{\left(-\sigma_{d}\right)^{\frac{1+2 n_{2}}{n_{2}}}}{\left(1+2 n_{2}\right) H_{2}^{\frac{1}{n_{2}}} E} \frac{2 n_{2}^{2}+n_{2}+1}{2\left(1+n_{2}\right)} \\
- & \left.-\frac{\left(-\sigma_{d}\right)^{\frac{2+n_{2}}{n_{2}}} n_{2}}{\left(2+n_{2}\right)\left(1+n_{2}\right) H_{2}^{\frac{2}{n_{2}}}}\right],
\end{aligned}
$$

where the unknown edge stress, $\sigma_{d}$, should be obtained by solving equation (16) with the help of the MatLab program system.

The $J$-integral solution in segment, $A_{2}$, of the integration contour (Fig. 1) coincides with (18). The components of the $J$-integral in segment $B$ of the integration contour (Fig. 1) were written as:

$$
\begin{gathered}
p_{x}=\sigma, \quad p_{y}=0, \\
d s=-d z_{3}, \quad \cos \alpha=1 .
\end{gathered}
$$

The coordinate, $z_{3}$, varies in the interval $[h,-h]$. The edge normal stress $\sigma_{a}$ in segment $B$ was determined from equation (16). For this purpose, $h$ and $\sigma_{d}$ were replaced by $2 h$ and $\sigma_{a}$, respectively. Besides, the bending moment in the beam cross-section ahead of the crack tip was written as $M=F a / 2$. Equation (12) was rewritten as:

$$
d z_{3}=\frac{h}{\varepsilon_{a}} d \varepsilon,
$$

where the edge linear strain $\varepsilon_{a}$ was expressed by the Ramberg-Osgood equation, i.e.:

$$
\varepsilon_{a}=\frac{\sigma_{a}}{E}+\left(\frac{\sigma_{a}}{H_{2}}\right)^{\frac{1}{n_{2}}} .
$$

Formulae (9), (11), (19), (20), (21) and (22) were substituted in (2). The integration was carried-out in boundaries from the lower edge normal stress to 
the upper edge normal stress. In this way, we obtained:

$$
\begin{aligned}
& J_{B}=-\frac{h}{\left(\frac{\sigma_{a}}{E}+\frac{\sigma_{a}^{\frac{1}{n_{2}}}}{H_{2}^{\frac{1}{n_{2}}}}\right)}\left[\frac{\sigma_{a}^{3}}{3 E^{2}}+\frac{\sigma_{a}^{\frac{1+2 n_{2}}{n_{2}}}}{\left(1+2 n_{2}\right) H_{2}^{\frac{1}{n_{2}}} E} \frac{2 n_{2}^{2}+n_{2}+1}{2\left(1+n_{2}\right)}\right. \\
&+ \frac{\sigma_{a}^{\frac{2+n_{2}}{n_{2}}} n_{2}}{\left(2+n_{2}\right)\left(1+n_{2}\right) H_{2}^{\frac{2}{n_{2}}}}-\frac{\left(-\sigma_{a}\right)^{\frac{1+2 n_{2}}{n_{2}}}}{\left(1+2 n_{2}\right) H_{2}^{\frac{1}{n_{2}}} E} \frac{2 n_{2}^{2}+n_{2}+1}{2\left(1+n_{2}\right)} \\
&\left.-\frac{\left(-\sigma_{a}\right)^{\frac{2+n_{2}}{n_{2}}} n_{2}}{\left(2+n_{2}\right)\left(1+n_{2}\right) H_{2}^{\frac{2}{n_{2}}}}\right] .
\end{aligned}
$$

The $J$-integral final solution was obtained by substitution of (18) and (23) in (3) and taking into account that $J_{A_{2}}=J_{A_{1}}$, i.e.:

$$
\begin{aligned}
& \text { (24) } J=\frac{h}{\left(\frac{\sigma_{d}}{E}+\frac{\sigma_{d}^{\frac{1}{n_{2}}}}{H_{2}^{\frac{1}{n_{2}}}}\right)}\left[\frac{\sigma_{d}^{3}}{3 E^{2}}+\frac{\sigma_{d}^{\frac{1+2 n_{2}}{n_{2}}}}{\left(1+2 n_{2}\right) H_{2}^{\frac{1}{n_{2}}} E} \frac{2 n_{2}^{2}+n_{2}+1}{2\left(1+n_{2}\right)}\right. \\
& +\frac{\sigma_{d}^{\frac{2+n_{2}}{n_{2}}} n_{2}}{\left(2+n_{2}\right)\left(1+n_{2}\right) H_{2}^{\frac{2}{n_{2}}}}-\frac{\left(-\sigma_{d}\right)^{\frac{1+2 n_{2}}{n_{2}}}}{\left(1+2 n_{2}\right) H_{2}^{\frac{1}{n_{2}}} E} \frac{2 n_{2}^{2}+n_{2}+1}{2\left(1+n_{2}\right)} \\
& \left.-\frac{\left(-\sigma_{d}\right)^{\frac{2+n_{2}}{n_{2}}} n_{2}}{\left(2+n_{2}\right)\left(1+n_{2}\right) H_{2}^{\frac{2}{n_{2}}}}\right] \\
& -\frac{h}{\left(\frac{\sigma_{a}}{E}+\frac{\sigma_{a}^{\frac{1}{n_{2}}}}{H_{2}^{\frac{1}{n_{2}}}}\right)}\left[\frac{\sigma_{a}^{3}}{3 E^{2}}+\frac{\sigma_{a}^{\frac{1+2 n_{2}}{n_{2}}}}{\left(1+2 n_{2}\right) H_{2}^{\frac{1}{n_{2}}} E} \frac{2 n_{2}^{2}+n_{2}+1}{2\left(1+n_{2}\right)}\right. \\
& +\frac{\sigma_{a}^{\frac{2+n_{2}}{n_{2}}} n_{2}}{\left(2+n_{2}\right)\left(1+n_{2}\right) H_{2}^{\frac{2}{n_{2}}}}-\frac{\left(-\sigma_{a}\right)^{\frac{1+2 n_{2}}{n_{2}}}}{\left(1+2 n_{2}\right) H_{2}^{\frac{1}{n_{2}}} E} \frac{2 n_{2}^{2}+n_{2}+1}{2\left(1+n_{2}\right)} \\
& \left.-\frac{\left(-\sigma_{a}\right)^{\frac{2+n_{2}}{n_{2}}} n_{2}}{\left(2+n_{2}\right)\left(1+n_{2}\right) H_{2}^{\frac{2}{n_{2}}}}\right] \text {. }
\end{aligned}
$$


The fact, that at linear-elastic behaviour, the $J$-integral value is equal to the strain energy release rate was used here to verify (24). For this purpose, by substitution of $\mathrm{H}_{2} \rightarrow \infty$ in (24), we obtained:

$$
J=\frac{9 F^{2} a^{2}}{16 E b^{2} h^{3}} .
$$

Expression (25) coincides with the formula for the strain energy release rate in the ENF beam configuration [22], which verifies (24). It should be mentioned, that at $E \rightarrow \infty$ and $n_{2}=1$, equation (24) transforms also in (25), which is another verification of the $J$-integral non-linear solution, obtained in the present study. The correctness of (24) is confirmed also by the fact, that at $n_{2}=1$ equation (24) transforms in (25).

The influence was evaluated of the material constants $n_{2}$ and $H_{2}$ (refer to equation (1)) on the non-linear fracture in the ENF configuration. For this purpose, formula (24) was used to calculate the $J$-integral value at different magnitudes of $n_{2}$ and $H_{2} / E$. In these calculations, it was assumed, that $b=$ $0.025 \mathrm{~m}, h=0.0015 \mathrm{~m}$ and $l=0.04 \mathrm{~m}$. The results of these calculations of $J$-integral are presented graphically in Fig. 5 (the $J$-integral is normalized by using the formula $\left.J^{N}=J /(E b)\right)$. The diagrams in Fig. 5 indicate, that the $J$-integral value decreases, when $n_{2}$ increases. It can be observed also, that the $J$-integral value decreases, when the ratio, $\frac{\mathrm{H}_{2}}{E}$, increases (Fig. 5).

The effect of the crack length on the $J$-integral value was analyzed. For this purpose, the $J$-integral was calculated at different crack lengths. The results obtained are reported in Fig. 6. The diagram clearly indicates that the $J$-integral value increases, when the crack length increases (Fig. 6).

\section{Conclusion}

Non-linear analysis was performed of fracture in the ENF beam configuration with taking into account the material nonlinearity. For this purpose, the Ramberg-Osgood stress-strain curve was used. The fracture behaviour was studied by applying the $J$-integral approach. Solutions of the $J$-integral are obtained by using a model based on the Classical beam theory. The influence of the material parameters in the Ramberg-Osgood equation on the $J$-integral value was evaluated. The effect of the crack length on the $J$-integral value was analyzed. It should be mentioned, that the analytical approach applied here is very useful for parametric analyses, since the simple formulae obtained capture the essential of the non-linear fracture. The analytical solutions, obtained in the present paper, can be applied also for calculation of the critical value of 


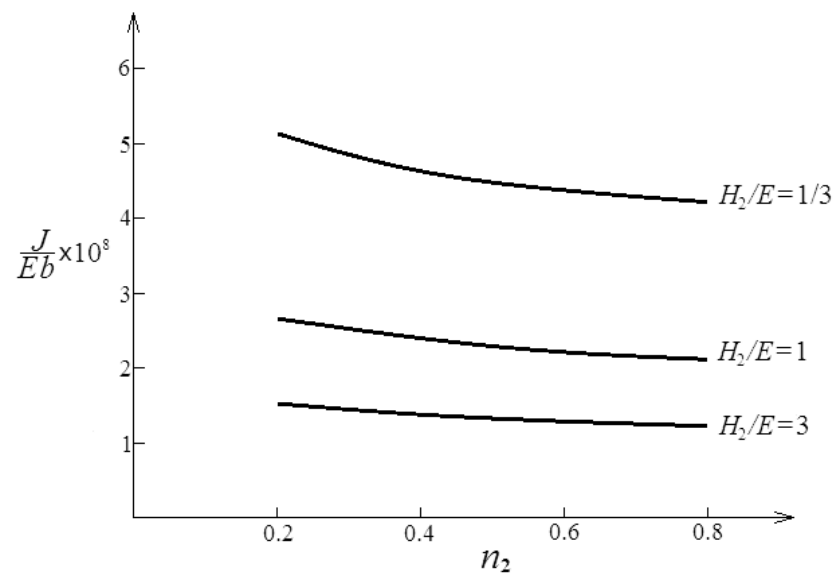

Fig. 5. Normalized $J$-integral plotted against $n_{2}$ at different values of the ratio $H_{2} / E$. The crack length is $a=0.5 l$

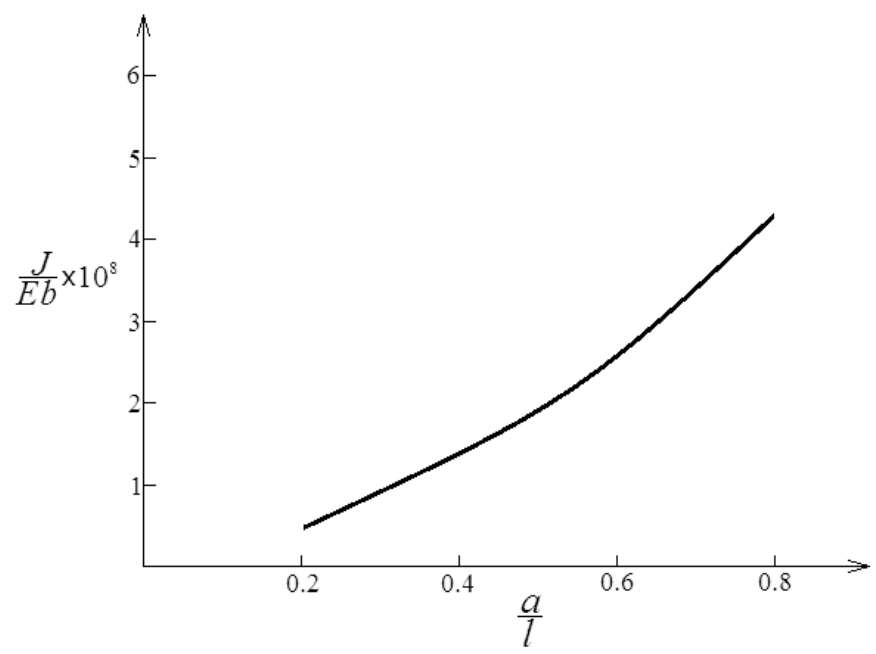

Fig. 6. Normalized $J$-integral plotted against the ratio crack length-to-half span, $a / l$, at $n_{2}=0.5, H_{2} / E=2$

the $J$-integral, with taking into account the material non-linearity by using the experimentally determined critical fracture load at the onset of crack growth from the initial crack tip position in the ENF configuration. 


\section{REFERENCES}

[1] Lukkasen, D., A. Meidell. Advanced Materials and Structures and their Fabrication Processes, HiN, Narvik University College, 2003.

[2] Apostolov, R., V. Kantchev, D. Dinev. On Some Peculiarities in the Deformation of Three - point Bended Sandwich Beam, Proceedings of 11-th National Congress on Theoretical and Applied Mechanics, 2-5 September, Bulgaria, Borovets, 2009.

[3] Dinev, D. Finite Element for Analysis of Beams Strengthened by Bonded Composite Strips. Engineering Mechanics - International Journal for Theoretical and Applied Mechanics, 18 (2011), No. 1, 51-63.

[4] Markov, I., D. Dinev. Theoretical and Experimental Investigation of a Beam Strengthened by Bonded Composite Strip, Reports of International Scientific Conference VSU'2005, 26-27 May, 2005.

[5] Ivanov, Ya., V. Stoyanov. High Technologies and New Construction Materials in Civil Engineering, In: Education, Science, Innovations (Proc. Ist Int. Conf. of the European Polytechnical University, June 9-10, 2011, Pernik, Bulgaria), European Polytechnical University, 2012, 161-169. (ISSN: 1314-5711)

[6] Serafimova, E., S. Milenkova, V. Stoyanov, J. Pelovsky, V. Petkova. Mechanical Testing of Ceramic Composite Materials, Proc. of the XV International scientific conference VSU'2015, June 4-5, Sofia, 2 (2015), 465-461.

[7] Bolotin, V. V. Delaminations in Composite Structures: its Origin, Buckling, Growth and Stability, Composites: Part B, 27B (1996), 129-145.

[8] Schon, J., T. Nyman, A. Blom, H. Ansell. Numerical and Experimental Investigation of a Composite ENF-specimen. Eng. Fract. Mech., 65 (2000), 405433.

[9] Carlsson, L. A., J. W. Gillespie, B. R. Trethewey. Mode II Interlaminar Fracture of Graphite/Epoxy and Graphite/PEEK. Journal of Reinforced Plastics and Composites, 5 (1986), 170-187.

[10] Hashin, Z., D. Bagchi, B. W. Rosen. Non-linear Behaviour of Fiber Composite Laminates, Report for NASA, Washington D. C., 1974.

[11] Lin, W.-P., H.-T. Hu. Nonlinear Analysis of Fiber-Reinforced Composite Laminates Subjected to Uniaxial Tensile Load. Journal of Composite Materials, 36 (2002), No. 12, 1429-1450.

[12] Hung, S.-C., K. M. Liechti. Nonlinear Multiaxial Behaviour and Failure of Fiber-Reinforced Composites, Time Dependent and Nonlinear Effects in Polymers and Composites, ASTM STP 1357, R. A. Schapery and C. T. Sun, Eds, American Society for Testing and Materials, PA, West Conshohocken, 2000, 176-222.

[13] Gates, T. S., J. L. Chen, C. T. Sun. Micromechanical Characterization of Nonlinear Behaviour of Advanced Polymer Matrix Composites, NASA Technical Memorandum 109129, Virginia, Hampton, Langley Research Center, 1994.

[14] Liv, A. Mechanics and Mechanisms of Fracture: an Introduction, ASM International, 2005. 
[15] Suemasu, H. Double Notched Split Cantilever Test Method to Measure the Mixed Mode II and III Interlaminar Toughness, 17-th International Conference on Composite Materials, UK, Edinburgh, 2009.

[16] De Morais, A., A. Pereira. Mixed Mode II+III Interlaminar Fracture of Carbon/Epoxy Laminates. Composites Science and Technology, 68 (2008), 20222027.

[17] Szekrenyes, A., J. UJ. Application of Elastic Foundation Beams in Composite Fracture Mechanics - Analysis of the ELS Specimen, In: Proceedings of the Fourth Conference on Mechanical Engineering, Springer Hungarica, Budapest, I (2004), 191-195.

[18] Szekrenyes, A., J. UJ. Comparison of Some Improved Solutions for Mixedmode Composite Delamination Coupons. Composite Structures, 72 (2006), 321329.

[19] Szekrenyes, A. Interlaminar Fracture Analysis in the $\mathrm{G}_{I^{-}} \mathrm{G}_{I I}-\mathrm{G}_{I I I}$ Space Using Prestressed Transparent Composite Beams. Journal of Reinforced Plastics and Composites, 30 (2011), No. 19, 1655-1669.

[20] Blackman, B. R. K., A. J. Kinloch. Fracture Tests for Structural Adhesive Joints, in Fracture Mechanics Testing Methods for Polymers, Adhesives and Composites, Eds. A. Pavan, D. R. Moore and J. G. Williams, Elsevier Science Ltd., UK, Oxford, 2001.

[21] Martin, R. H. Interlaminar Fracture Characterization. Key Eng. Mater., (1996), $121-122$.

[22] Rikards, R., A. Korjakin, F.-G. Buchholz, H. Wang, A. Bledzki, G. WACKER. Interlaminar Fracture Toughness of GFRP influenced by Fiber Surface Treatment. Journal of Composite Materials, 32 (1998), No. 17, 1528-1559.

[23] Dowling, N. Mechanical Behaviour of Materials, 2007.

[24] Rice, J. R. A Path Independent Integral and the Approximate Analysis of Strain Concentration by Notches and Cracks. Journal of Applied Mechanics, 35 (1968), 379-386.

[25] Cherepanov, G. Brittle Materials Fracture Mechanics, Moscow, Nauka, 1974.

[26] Broek, D. Elementary Engineering Fracture Mechanics, Springer 1986.

[27] Hoff, N. J. The Analysis of Structures, New York, John Wiley\&Sons, 1956.

[28] NADAI, A. Theory of Flow and Fracture of Solids, 2, New York, McGraw-Hill, 1963.

[29] Seely, F. B., J. O. Smith. Advanced Mechanics of Materials, New York, John Wiley\&Sons, 1967.

[30] Washizu, K. Variational Methods in Elasticity and Plasticity, Oxford, Pergamon Press, 1974.

[31] Malinin, N. N. Applied Theory of Plasticity and Creep, Moscow, Mashinostroenie, 1968. 